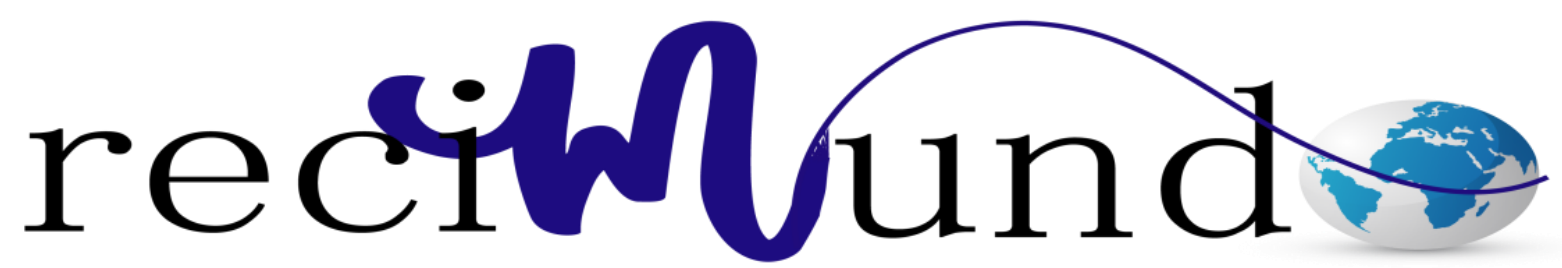

Revista Científica Mundo de la Investigación y el Conocimiento

Verónica Natalia Maroto Hidalgo ${ }^{\text {a }}$; Helen Verónica Veas García ${ }^{\text {b; }}$ Andrea Dolores Ordoñez Balladares ${ }^{c}$; Diego Omar Loza Jarama ${ }^{\mathrm{d}}$

Patologías y manifestaciones bucodentales producidas por cáncer

y tratamientos en niños

Pathologies and oral manifestations produced by cancer and treatments in children

Revista Científica Mundo de la Investigación y el Conocimiento. Vol. 2 núm.3, julio, ISSN: 2588-073X, 2018, pp. 95-110

DOI: 10.26820/recimundo/2.(3).julio.2018.95-110

Editorial Saberes del Conocimiento

Recibido: 05/04/2018

Aceptado: 15/05/2018

Publicado: 30/07/2018

Correspondencia: verónica.marotoh@ug.edu.ec

a. Universidad de Guayaquil; verónica.marotoh@ug.edu.ec

b. Universidad de Guayaquil; helen.veasgarcia@ug.edu.ec

c. Universidad de Guayaquil; andrea.ordonezb@ug.edu.ec

d. Universidad de Guayaquil; diego.lozaj@ug.edu.ec 
Patologías y manifestaciones bucodentales producidas por cáncer $\mathbf{y}$ tratamientos en niños

Vol. 2, núm. 3., (2018)

Verónica Natalia Maroto Hidalgo; Helen Verónica Veas García; Andrea Dolores Ordoñez Balladares; Diego Omar Loza Jarama

\section{RESUMEN}

El cáncer es una enfermedad que hoy en día afecta a muchos menores, de tal manera que el cáncer infantil tiene características propias, considerándolo así , como una gran consecuencia de muerte en menores de 14 años, tal demuestra los estudios realizados. Siendo así con una rango de edad entre los o -14 se puede detectar en niños los distintos canceres, los más comunes son las leucemias, cáncer de encéfalos y otro tumores pertenecientes al SNC.

Aunque es considerado como una enfermedad mortal esta se puede tratar, lo cual sus tratamientos consisten en quimioterapias y radioterapias, estos son los más comunes a la hora de realizar un tratamiento en un niño. Es claro decir que estos tratamientos sirven de mucha ayuda pero a la vez tiene sus complicaciones en el paciente pediátrico. Estos tipos de perecimientos que se implementan a los menores tienen sus efectos tardíos que pueden recaer en cualquier parte del cuerpo, sobre todo en la cavidad bucal, especialmente cuando se está tratando la leucemia.

Es por eso que esta investigación se enfatiza en explicar todas las manifestaciones que se puedan presentar en la cavidad bucal, tras un tratamiento para el cáncer. Además muestra tanto como el odontólogo y el paciente, en el caso del odontólogo como debe llevar la situación frente a una neoplasia y de la misma manera como el paciente debe mantener su cavidad bucal en una óptima higiene para evitar mayores complicaciones.

Palabras claves: Cáncer; infantile; quimioterapia; radioterapia. 


\title{
Patologías y manifestaciones bucodentales producidas por cáncer y \\ tratamientos en niños
}

Vol. 2, núm. 3., (2018)

Verónica Natalia Maroto Hidalgo; Helen Verónica Veas García; Andrea Dolores Ordoñez

Balladares; Diego Omar Loza Jarama

\begin{abstract}
Cancer is a disease that today affects many children, so that childhood cancer has its own characteristics, considering it as a great consequence of death in children under 14 years, as shown by the studies carried out. Thus, with an age range between $0-14$, different cancers can be detected in children, the most common being leukemia, cancer of the brain and other tumors belonging to the CNS.
\end{abstract}

Although it is considered a deadly disease it can be treated, which treatments consist of chemotherapies and radiotherapies, these are the most common when it comes to a treatment in a child. It is clear to say that these treatments are very helpful but at the same time have its complications in the pediatric patient. These types of deaths that are implemented to minors have their late effects that can fall on any part of the body, especially in the oral cavity, especially when treating leukemia.

That is why this research is emphasized in explaining all the manifestations that may occur in the oral cavity, after a treatment for cancer. It also shows as much as the dentist and the patient, in the case of the dentist how it should take the situation in front of a neoplasm and in the same way as the patient must maintain his oral cavity in optimal hygiene to avoid further complications.

Keywords: Cancer; infant; chemotherapy; radiotherapy. 
Patologías y manifestaciones bucodentales producidas por cáncer $\mathbf{y}$ tratamientos en niños

Vol. 2, núm. 3., (2018)

Verónica Natalia Maroto Hidalgo; Helen Verónica Veas García; Andrea Dolores Ordoñez Balladares; Diego Omar Loza Jarama

\section{Introducción.}

El presente artículo se centra en conocer la interacción de la cavidad oral con la presencia de cáncer en infantes, de tal manera que sé que se lo considera un entorno adecuado para la consecuencias radicales de dicha enfermedad e incluso se tiene en cuenta las consecuencias tanto directos e indirectos de la quimioterapia. Aunque presuntamente los cambios en las estructuras del tejido blando de la cavidad oral reflejen los cambios que ocurren en todo el aparato gastrointestinal, la presente investigación se concentrará en las complicaciones orales provocadas por la terapia con antineoplásicos y por la radioterapia.

Puesto que el tema a tratar, abarca ciertas manifestaciones orales provocadas por el deterioro inmunológico del paciente antes y durante su tratamiento antineoplásico, se requiere de un diagnóstico con una minuciosa historia clínica, exploración física y estudios de laboratorio que la confirmen. Es por eso que se considera este tema de mucha importancia para las personas debido a que pueden conocer que trae consigo en cáncer en relación a la cavidad bucal y también cual es prevención y sobre todo el pro y contras de los tratamientos.

Es por esto que este artículo tiene como objetivo determinar las patologías y manifestaciones bucodentales que se pueden presentar. De la misma manera en analizar cuáles son los factores que ocasionan que la aparición de las manifestaciones bucodentales en niños. Y finalmente en interpretar los diferentes tratamientos para las patologías que se puedan presentar en los niños. 


\section{Patologías y manifestaciones bucodentales producidas por cáncer y \\ tratamientos en niños}

Vol. 2, núm. 3., (2018)

Verónica Natalia Maroto Hidalgo; Helen Verónica Veas García; Andrea Dolores Ordoñez

Balladares; Diego Omar Loza Jarama

\section{Desarrollo.}

Se considera al cáncer como una enfermedad en el cual observa su objetividad desde tiempos añejos. En los últimas décadas hemos escuchado más acerca de una terrible enfermedad denominada cáncer, esta palabra al escuchar asusta a muchos, y claro está, debido a que la padece no solo adultos, si no también cientos de niños que mueren lamentablemente a causa de esta enfermedad. Realmente, el cáncer consiste en el deterioro de las células, siendo estas las unidades estructurales de los seres vivos ${ }^{1-2}$.

El cáncer en los niños o cáncer infantil, se muestra con una constancia leve o menor, ya sea entre las enfermedades pediátricas o entre las enfermedades neoplásicas o cáncer de la población universal, sin embargo constituye la segunda causa más frecuente de muerte en niños mayores de un año. Los analistas revelan una incidencia anual de 120 nuevos casos de menores de 15 años, el cáncer no es selectivo, este puede padecer cualquier menor si medir su edad, sexo, raza y sitio geográfico ${ }^{4-3}$.

El cáncer aparece cuando las celular anormales crecen y se disperse velozmente, de tal manera que las células normales que el cuerpo posee, aumentan, se fraccionan y son capaces de parar su aumento. Además con el tiempo estas empiezan a perecer. Mientras que estas células normales, las células cancerosas continúan en desarrollo y fraccionándose de manera desmandada y no se mueren cuando estas tienen la responsabilidad de hacerlo ${ }^{1}$.

Realizando un análisis se llega a la conclusión que en los últimos cuatro años aparecen más de diez patologías más habituales con manifestaciones estomatológicas, que solicitan una 


\section{Patologías y manifestaciones bucodentales producidas por cáncer y tratamientos en niños}

Vol. 2, núm. 3., (2018)

Verónica Natalia Maroto Hidalgo; Helen Verónica Veas García; Andrea Dolores Ordoñez Balladares; Diego Omar Loza Jarama

mayor preparación, tales como, ciertas infecciones por caries en una paciente infante con leucemia, por tal motivo, dicho paciente está más propenso o necesitado de solicitar una excelente calidad de cuidado y compromiso ${ }^{5}$.

Al momento de presentarse las manifestaciones estomatológicas en el caso del cáncer de le leucemia aguda lifoblastica y mieloblastica, en estas se puede presentar manifestaciones de un primer grado o primarias, que claramente son propias de dicha enfermedad, las del segundo grado o simplemente secundarias, estas son las secuelas que deja el tratamiento, y por último están las terciarias, en el cual están presenten en este grado, las infecciones oportunistas ${ }^{5}$. Por lo tanto a consecuencia del cáncer, las manifestaciones que ocurren en la cavidad bucal son las petequias, las equimosis, la xerostomía, la candidiasis herpes, el infiltrado leucocitario y los trastornos de la deglución ${ }^{5-6}$.

El cáncer infantil en las últimas décadas posee una exorbitante significación en el entorno infantil. El cáncer afecta mayormente a niños que a niñas. El niño afectado o enfermo por un tumor maligno, tiene la capacidad de poderse desarrollar y crecer muy altos, a diferencia del adulto. Por lo consiguiente la transformación normal del crecimiento, se observa fuertemente lesionado por la enfermedad y por el tratamiento que el paciente pediátrico se llevará a cabo ${ }^{7}$.

Los tumores o cáncer que son típicos en los infantes son los denominados "tumores sólidos" lo cual esto ocurre en un rango que va por debajo de los 5 años de vida. Asimismo sus localizaciones anatómicas, no afectan a epitelios, que no van a producir hemorragias superficiales, ni exfoliación de células tumorales. En sí, estos no van a perjudicar órganos con superficies exhibas o expuestas como son la piel, intestino y el pulmón ${ }^{8-9}$. 


\section{Patologías y manifestaciones bucodentales producidas por cáncer y \\ tratamientos en niños}

Vol. 2, núm. 3., (2018)

Verónica Natalia Maroto Hidalgo; Helen Verónica Veas García; Andrea Dolores Ordoñez

Balladares; Diego Omar Loza Jarama

Se considera que de todas las neoplasias infantiles, las más frecuentes son las leucemias que forman parte 1/3 del total (de ellas, más del $80 \%$ son leucemias linfoblásticas agudas) y cerca de la mitad de todos los cánceres en la infancia, si se consideran conjuntamente las leucemias y los linfomas ${ }^{10}$.

El resto de neoplasias infantiles: neuroblastomas (8\%), tumor de Wilms (7\%), sarcomas de partes blandas (6\%), tumores óseos (5\%), retinoblastomas (3\%), hepatoblastomas (2-3\%) o tumores de células germinales, aun siendo menos frecuentes son característicos, porque prácticamente sólo se encuentran en la infancia ${ }^{11}$.

La determinación para las posibles causas del cáncer infantil, no logran dar un concepto o análisis concreto, sin embargo se conoce que la mayoría de los canceres infantiles tienen una etiología multifactorial, causado por una mutación genética hereditaria, que provocan un desarrollo celular desmandado y finalmente cáncer. Igualmente estudios han comprobado que la exposición a la radiaciónionizante, logra perjudicar el ADN, con el fin de que el menor presente leucemia y posiblemente otros tipos de cáncer ${ }^{12-13}$.

El cáncer infantil no solo es considerado como una enfermedad, al contrario incorpora un mayor número de enfermedades, que traen consigo características particulares. No obstante, la mayoría de las enfermedades tienen en común que se provocan a partir del desarrollo anormal de una única célula o de un conjunto de ellas, que adquieren la capacidad de involucrar tanto los órganos vecinos como los alejados ${ }^{13}$. 


\section{Patologías y manifestaciones bucodentales producidas por cáncer y tratamientos en niños}

Vol. 2, núm. 3., (2018)

Verónica Natalia Maroto Hidalgo; Helen Verónica Veas García; Andrea Dolores Ordoñez Balladares; Diego Omar Loza Jarama

Es relativo que los tumores malignos infantiles son con frecuencia de cabeza y cuello, incluyendo el SNC y órganos linfoides, a pesar de que el cáncer tenga su ubicación alejada de la zona maxilofacial, el tratamiento, tanto la quimioterapia como la radioterapia traen consigo una acción violenta y sistémica en un organismo que se encuentra en pleno crecimiento. La presencia de las manifestaciones orales severas, al relacionarse con paciente que están en un desarrollo y crecimiento de todos sus órganos, incluyendo la dentición y estructuras fáciles. Suelen observase ciertas manifestaciones o anomalíasóseas, de esmalte, malformaciones dentarias a nivel de raíces, coranas y presencia de dientes rudimentarios. Ocasionado al tipo de tratamiento que recibe el paciente. Con fundamentos científicos se ha demostrado que las caries, patologías gingivales y maloclusiones se incrementan con la edad, de la misma manera que sucede en $\operatorname{personas~sanas~}^{14}$.

Existen enfermedades comunes y enfermedades inmunitarias que ocasionan ciertas lesiones en la mucosa bucal, una de las principales enfermedades que produce estas manifestaciones en la cavidad oral son las leucemias .los pacientes con esta enfermedad presentan manifestaciones en su cavidad oral, de tal manera que el papel funcional del odontólogo en los principio del cáncer, es de mucha ayuda e importancia ${ }^{15}$.

Las manifestaciones bucales ocasionadas por las Leucemias tienen una clasificaciones muy relevante, en primarias; Las cuales corresponden al daño o deterioro de los tejidos por el efecto de la enfermedad, además llegan aparecer alteraciones periodontales (inflamación de las células en el corion gingival), con respecto a las alteraciones en la mucosa (deterioro de la encía), por último se presente las alteraciones radiográficas ( muestra a nivel radiográfico los cambio 


\section{Patologías y manifestaciones bucodentales producidas por cáncer y \\ tratamientos en niños}

Vol. 2, núm. 3., (2018)

Verónica Natalia Maroto Hidalgo; Helen Verónica Veas García; Andrea Dolores Ordoñez

Balladares; Diego Omar Loza Jarama

que se presentan en la cavidad bucal tales como ausencia de conductos, destrucción de la lámina dura entre otros) $)^{16}$.

A nivel segundario, corresponde a las lesiones ocasionadas por la quimioteria, además resulta de una interacción complicada con diversos factores tales como: mucositis (inflamación y ulceración de la mucosa), xerostomía (alteración del flujo salival), infecciones (ocasionado por la eliminación de la medula ósea), y por último encontramos en la hemorragia (ocasionada por la plaquetopenia) ${ }^{17-16}$.

Los problemas orales son complicaciones comunes de tratamientos contra el cáncer y son muy frecuentes en los pacientes en la fase terminal de su enfermedad. Manifestando la variedad y complejidad de signo y síntomas que se puede presentar y resumir que los riesgos primordiales de los leucémicos son la hemorragia y la infección. El tratamiento adecuado consiste en prevenir que estas complejidades se presenten ${ }^{18}$.

La intervención del odontólogo, se debe realizar inmediatamente, con anterioridad al tratamiento oncológico, en el transcurso del tratamiento médico y posterior del mismo. De tal manera que se debe plantear una excelente historia clínica y exploración bucal con todos los parámetros indicados ${ }^{18-19}$.

Paciente con leucemia aguada: interviene el cirujano dentista debido a que aparecen con frecuencia infecciones, ulceras. El odontólogo da el diagnostico correspondiente guiándose por los números de eritrocitos y plaquetas. De la misma manera el paciente leucémico en receso: el odontólogo, debe asegurase que los nivele de plaquetas y eritrocitos estén normales para el 


\section{Patologías y manifestaciones bucodentales producidas por cáncer y tratamientos en niños}

Vol. 2, núm. 3., (2018)

Verónica Natalia Maroto Hidalgo; Helen Verónica Veas García; Andrea Dolores Ordoñez Balladares; Diego Omar Loza Jarama

manejo del paciente. Así mismo el paciente leucémicos en fase de remisión: el odontólogo elimina los irritantes locales y reducir los riesgos infecciosos.

Uno de los tratamientos por el cáncer es la quimioterapia en el cual consiste en medicamentos que se base es destruir las células cancerosas. Sin embargo estos medicamentos sé que reciben durante la quimioterapia pueden ocasionar efectos secundarios en la cavidad bucal, puede ser graves, aunque a simple viste se vean inofensivos, la cual incluyen problemas en los diente, encías tejidos blandos y glándulas salivales. La mayoría de los problemas ocurren únicamente durante la fase del tratamiento o incluso un tiempo después que este termine ${ }^{20}$.

Como consecuencia de los efectos secundarios estos ocasionan dolor e incluso que el comer, tragar y hablar sea muy dificultoso. De cierta manera a los pacientes que están con este tratamiento son más propensos a obtener infecciones bucales, y por ultimo si se detecta que los efectos secundarios son graves, el paciente tiene una probabilidad de no seguir con el tratamiento hasta que el médico lo indique.

Los pacientes sometidos a ciclos terapéuticos de quimioterapia desarrollan alteraciones en la mucosa tan graves que se corre el peligro de obtener situaciones septicémicas graves, difíciles de controlar a nivel farmacológico, sobre todo en pacientes no sometidos preventivamente a limpieza de la cavidad oral. La mucosa oral puede alterarse por la patología presente así como los tratamientos indicados para erradicarlas ${ }^{20}$.

A diferencia de la anterior, la radioterapia consiste una alternativa muy relevante en el tratamiento de las lesiones, pero las consecuencias de la radiación no solo perjudican las células 


\section{Patologías y manifestaciones bucodentales producidas por cáncer y \\ tratamientos en niños}

Vol. 2, núm. 3., (2018)

Verónica Natalia Maroto Hidalgo; Helen Verónica Veas García; Andrea Dolores Ordoñez

Balladares; Diego Omar Loza Jarama

malignas, en cambio que esta es así mismo absorbida por los tejidos bucales y peribucales sanos.

Las manifestaciones de efectos luego de la utilización de la radioterapia constituye la principal limitación de la misma, pese al empleo desarrollo de habilidades que tantea renovar el índice terapéutico $^{21-22}$.

En efecto puede presentarse lesiones bucales antes y posteriormente a la radiación, efectos que se han nombrado anteriormente como son la mucositis, xerostomía disgeusia, ageustia, infecciones, caries, periodontitis. La radioterapia logra destruir directamente y aislar el tejido de la boca, las glándulas salivales y el hueso ${ }^{23}$.

Estas laceraciones reducen la calidad de vida de estos pacientes y motivan a interceptar en la prolongación de la radioterapia, exponiendo en riesgo su vida. Considerando la importancia de incorporar a estos pacientes en protocolos de atención odontológica preventiva, en que se desarrollen un examen bucal personalizado que advierta la obligación del tratamiento y posteriores verificaciones periódicas que protejan las medidas preventivas adoptadas, siempre en conjunto con el oncólogo tratante.

Luego de la terapia oncológica hay que tener presente las medidas de higiene conocidas, controlar el estado de salud oral y la eficacia de las restauraciones ya realizadas, y realizar el tratamiento adecuado para alguna patología que se pueda diagnosticar. Los pacientes sometidos a QT, RT o ambos pueden presentar cansancio, caquexia, depresión, lo cual puede provocar que no siga los protocolos de higiene oral ideados. El paciente debe conocer que la higiene oral sistemática y rutinaria puede reducir la incidencia y la severidad de las secuelas orales de la terapia contra el cáncer y motivarlos en su compromiso ${ }^{24}$. 
Patologías y manifestaciones bucodentales producidas por cáncer $\mathbf{y}$ tratamientos en niños

Vol. 2, núm. 3., (2018)

Verónica Natalia Maroto Hidalgo; Helen Verónica Veas García; Andrea Dolores Ordoñez Balladares; Diego Omar Loza Jarama

Debe plantearse un buen protocolo de atención odontológica antes a la terapia oncológica para evitar la realización de exodoncias postradiación. Caso contrario, hay que evitar hacer exodoncias entre los 2 meses y un año luego de la radiación por el riesgo de ORN. Si el paciente no puede esperar tanto tiempo se debe tomar en cuenta el tratamiento que le indique el médico ${ }^{24}$.

Los odontopediatras cumplen un rol fundamental en la prevención y tratamiento de los problemas bucales que puedan involucrar la salud del paciente pediátrico oncológico, de tal manera que debe integrar y capacitarse en grupos multidisciplinarios. Además se recomienda que el paciente se realice un examen odontológico íntegro y tratamiento siendo esto parte de un reglamento del tratamiento pre-cáncer ${ }^{25}$.

Es de mucha significación que tanto el odontólogo y el oncólogo se comuniquen entre ellos antes del que paciente empiece algún tratamiento para el cáncer. Es necesario que el paciente visite al odontólogo al menos un mes con anterioridad de empezar el tratamiento, de tal manera que el dentista realizara un examen completo de la cavidad bucal, dando así indicaciones para cuando el paciente ya se encuentre en el proceso de su tratamiento, este deberá revisar su boca diariamente para constatar si existen heridas o si han producido otros cabios ${ }^{25}$.

En caso de que el paciente muestre signos de inflamación en la boca es recomendable que tome mucha agua, chupe trozos de hielo, use goma de mascar sin azúcar y la implementación de sustitutos salivales para mantener la boca húmeda. Por otro lado, para que el paciente mantenga sus cavidad bucal en optima condiciones, es necesario que se cepille la boca con un cepillo blando desde de cada comida. Utilización de pasta con flúor, es adecuado que al memento de utilizar los enjuagues bucales, éstos no contengan alcohol. Además el uso del hilo 


\section{Patologías y manifestaciones bucodentales producidas por cáncer y \\ tratamientos en niños}

Vol. 2, núm. 3., (2018)

Verónica Natalia Maroto Hidalgo; Helen Verónica Veas García; Andrea Dolores Ordoñez

Balladares; Diego Omar Loza Jarama

dental y de realizase un enjuague con bicarbonato, sal y agua, es indispensable para mantener limpia la boca ${ }^{26}$.

Es importante recordar, que el paciente con cáncer debe visitar a su dentista antes de comenzar cualquier tratamiento ya sea QTORT, además de tener un cuidado especial con la boca durante el tratamiento, y es considerable tener un comunicación regular con ambos médicos odontólogo y oncólogo acerca de cualquier problema que se presente durante y después de los tratamientos antes mencionados ${ }^{27}$.

\section{Discusión.}

Esta investigación consiste en demostrar que ya siendo el cáncer una enfermedad con un índice alto en mortalidad tiene una cura, que en algunas ocasiones logra su cometido a un 100\% y en otras no lo logra. Estos tratamientos que existen son de gran ayuda para la humanidad, dando la espereza de una salud óptima al paciente que padece de cáncer. Pero una gran desventaja de los procedimientos que se utilizan para la erradicación del cáncer puede ocasionar en la mayoría de los casos ciertas lesiones o efectos secundarios en el organismo del cuerpo humano.

Refiriéndose de la radioterapia, es requerido que antes del tratamiento se disponga una valoración odontológica pre-radiación, en el cual se examine la boca del paciente, endodóntico y de la misma manera a nivel periodontal. En el caso de la quimioterapia la asistencia se centra en faltar cierta eventualidad de infección al contrario sobretodo se dirige en aminorar la inmunosupresión que ocasiona el paciente. 
Patologías y manifestaciones bucodentales producidas por cáncer $\mathbf{y}$ tratamientos en niños

Vol. 2, núm. 3., (2018)

Verónica Natalia Maroto Hidalgo; Helen Verónica Veas García; Andrea Dolores Ordoñez Balladares; Diego Omar Loza Jarama

El tratamiento para el cáncer infantil perjudica el sistema inmune, incrementando el peligro de bacteriemia y septicemia. A manera la cavidad bucal consiste en una puerta de acceso de agentes patógenos al torrente sanguíneo, de tal modo que el tratamiento dental tiende a ser sustancial en estos niños. Además esta información busca informar a los padres o responsables sobre la enfermedad, del tratamiento de la misma, su alcance en la cavidad oral. También que los pacientes logren concientizar y responsables con los respectivos cuidados con la higiene de su cavidad bucal. Y finalmente impulsar el tratamiento odontológico anticipado a la quimioterapia o radioterapia para reducir el índice de complicaciones.

\section{Conclusiones.}

Finalmente como ya se ha remarcado con anterioridad el cáncer es una de las enfermedades que más afectan tanto a adultos, como también puede afectar en gran espectro a niños de cualquier edad. Se han demostrado por medio de los párrafos anteriormente leídos ciertas evidencias que el cáncer tiene una gran repercusión dentro de lo que se conoce cavidad oral especialmente en los infantes debido a su etapa de desarrollo y crecimiento.

Como ya se ha demostrado una de los grandes tipos de cáncer que puede provocar gran daño en los tejidos bucales es la leucemia debido al gran impacto que está puede llegar a causar dentro del infante. Se pueden dar recomendaciones para el cuidado bucal dentro de estos pacientes como él visitar a un Odontólogo cirujano, debido a las infecciones que se pueden causar debido al cáncer. También se puede llevar a cabo sesiones rutinarias con la respectiva quimioterapia o radioterapia para tener un control del cáncer en cuestión, con la desventajas que estas sesiones traen como consecuencias afecciones en la cavidad bucal. 


\section{Patologías y manifestaciones bucodentales producidas por cáncer y \\ tratamientos en niños}

Vol. 2, núm. 3., (2018)

Verónica Natalia Maroto Hidalgo; Helen Verónica Veas García; Andrea Dolores Ordoñez

Balladares; Diego Omar Loza Jarama

\section{Bibliografía.}

1. Kidshealth. [Internet]. Kidshealth. 2015. Disponibel en: https://kidshealth.org/es/kids/health-problems-esp/teeth-sp?ref=search\&WT.ac=msh-kdtop-es-search-clk

2. Garza Salazar J., Juárez, P. El cáncer.Monterrey: Universidad Autónoma de Nuevo León; 2014. Disponible en http://eprints.uanl.mx/3465/1/El_Cancer.pdf

3. Rubinstein EB, Miller WL, Hudson SV, Howard J, O'Malley D1 Tsui J, Lee HS, Bator A, Crabtree BF. Cancer survivorship care in advanced primary care practices:a qualitative study of challenges and opportunities. Jama Intern Med. 2017; 177(2):17261732

4. Cabrerizo MC, Oñate R. Aspectos Odontoestomatológicos en oncología infantil. Med Oral Patol Oral Cir Bucal. 2005 febrero; 10(1): 21-7

5. Teja-Ángeles de la E, Durán A, Espinosa L, Ramírez J. Manifestaciones estomatológicas de los trastornos sistémicos más frecuentes en el instituto nacional de Pediatría. Revisión de la literatura y estadísticas del instituto. Acta Pediatr Mex. 2008; 29(4):189-99

6. Nasim VS, Shetty YR, Hegde AM. Dental health status in children with acute lymphoblastic leukemia. J Clic Pediatr Dent. 2007; 31(3): 2010-3

7. Pacheco M, Madero L. Oncología Pediátrica. Rev Cient Complutense. 2003; 0(1):107116

8. Fajardo A. Mortalidad por cáncer en niños. Bol Med Hosp Infant Mex. 2005; 61(1):1-3

9. Gallego C, Martínz MJ, Guerrero N, Romemro I, García F, Manzano V. Manejo del dolor en el paciente oncológico pediátrico y factores que influyen en su percepción. Rev Cubana Farm. 2015; 49(2): 394-411

10. Ward E, DeSantis C, Robbins A, Kohler B, Jemal A. Chidhoof and adolescent cancer statistics 2014. CA: A Cancer Journal for Clinicians. 2014; 64(2): 79-82

11. Rebecca L. Siegel KDAJ. Cancer Statics 2018. CA: A Cancer Journal for Clinicians. 2018; 68(1): 50

12. Huerta A . Oncología para el pediatra de Atención Primaria. Oncología para el pediatra de Atención Primaria (I): signos y síntomas sugerentes de patología neoplásica. Form Act Pediatr Aten Prim. 2014;7:4-15.

13. Vargas L.Cáncer en pediatría.Aspectos generales. Rev. chil. pediatr.2007; 71(4): 283-95

14. Osorio A, Bermúdez S, Lambertini A, Guerra ME. Experiencia en educacion, prevencion y contro de complicaciones orales de niños con cáncer. Odontol Pediatr. 2015; 14(1):6-18

15. Alayón C, Rodríguez Y, González E, León R, Ayala N. Manifestacion paeridontales de la leucemia mieloide aguda: reporte de un caso. AMC. 2017; 21(3):384-92

16. Aro M, Barazarte J, Ramírez D, Rupiz G. Protocolo a seguir por el odontólogo para la 

Patologías y manifestaciones bucodentales producidas por cáncer $\mathbf{y}$
tratamientos en niños

Vol. 2, núm. 3., (2018)

Verónica Natalia Maroto Hidalgo; Helen Verónica Veas García; Andrea Dolores Ordoñez Balladares; Diego Omar Loza Jarama

atención de las manifestaciones bucales frecuentes en pacientes con leucemia. Universidad Nororiental Privada "Gran Mariscal de Ayacucho". 2012. Disponible en https://issuu.com/ugmadirecciondeinvestigacion/docs/protocolo_de_atenci_n_manifest acio

17. Castellanos J, Gay O, Díaz . Medicinia en Odontología: Manejos Dental de Paciente con Enfermedades sistémicas. In.: El manual Moderno; 2015.

18. Rivera L, Teja E, Durpan L. Manejo paliativo de manifestaciones estomatológicas en un paciente pediátrico con leucemia en etapa terminal. Reporte de caso Clinico. Acta Pediáatr de Méx. 2015 Marzo; 36(2): 97-104. Disponible en http://www.scielo.org.mx/scielo.php?script=sci_arttext\&pid=S018623912015000200007

19. Parra J, Alvarado MC, Monsalve P, Montesinos G. Condiciones de salud bucodental en niños menores de catro años con leucemia linfoblástica aguda, antes del tramamiento de quimioterapia en el Instituto del Cancer (SOLCA), Cuenca. Rev Odont. 2016; 28(3):194-203. Disponible en file:///C:/Users/HP/Downloads/202-358-1-PB.pdf

20. Ferreiro J, García J, Baerceló R, Rubio I.Quimioterapia: efectos secundarios. Gac Med Bilb.2003; 100(2):69-74

21. Montero A, Hervás A, Morera R, Sancho S, Córdoba S, Corona J, Rodríguez I, Chajón E, Ramos A. Control de síntomas crónicos. Efectos secundarios del tratamiento con radioterapia y quimioterapia. Oncología (Barc). 2010 Marzo; 28(3).

22. Cedeño M. JA,RN,T,RA. Manifestaciones Bucales de los pacientes sometidos a radioterapia en cabeza y cuello, pautas de atención odontológica.2005; 28(3):41-50

23. Hurtado D, Estrada J. Complicaciones orales en pacientes sometidos a radioterapia. Univ.Odontol. 2012; 31(67): 111-129.

24. Lanza D. Tratamiento odontologico integral del paciente Oncológico. Parte II. Odontoestomatología. 2013;15(22):46-63

25. Acosta de Camargo MG, Bolívar M, Giunta C. Manejo Odontológico de paciente pediátricos oncológicos. Revisión bibliográfica. Rev. Latinoama de Ortod Odontoped. 2015.

26. Instituto Nacional de Investigación Dental y Craneofacial. La Quimioterapia y la boca. Bethesda. Instituto Nacional de Investigación Dental y Craneofacial; 2012. Disponible en https://www.nidcr.nih.gov/sites/default/files/2017-09/chemotherapy-and-yourmouth-spanish.pdf

27. Santos J, Ventiades J, Fontana N, Miranda C. Conducto odontológica en pacientes pediátricos portadores de leucemia. Rev Cubana Estomatol. 2007; 4(4):1-12 